\title{
Emergency single-port laparoscopic partial adrenalectomy for adrenal abscess in an adult with disseminated Streptococcus pyogenes bacteraemia: a case report
}

DS Tam *, MB, BS, CH Man, FHKAM (Surgery), KW Wong, FHKAM (Surgery), KC Ng, FHKAM (Surgery)

Department of Surgery, Caritas Medical Centre, Hong Kong

* Corresponding author: dicksontamds@gmail.com

Hong Kong Med J 2021;27:52-4

https://doi.org/10.12809/hkmj208474

\section{Case report}

A 55-year-old man with unremarkable past health who was a chronic smoker and drinker presented to the emergency department in July 2019 with 2-day history of severe epigastric pain and fever. On admission, the patient was septic-looking and clinically unstable (blood pressure $138 / 94 \mathrm{~mm} \mathrm{Hg}$, pulse $115 \mathrm{bpm}$, temperature $39.2^{\circ} \mathrm{C}$ ). Physical examination revealed epigastric tenderness and guarding. Blood tests showed an elevated white cell count of $20.89 \times 10^{9} / \mathrm{L}$ (reference range, $3.70-9.20 \times 10^{9} / \mathrm{L}$ ) and $\mathrm{C}$-reactive protein level of $>294 \mathrm{mg} / \mathrm{L}$ (reference range, $<5.0 \mathrm{mg} / \mathrm{L}$ ). Chest and abdominal plain radiograph showed no free gas under the diaphragm. Urgent contrast computed tomography (CT) of the abdomen and pelvis demonstrated a $4.5 \mathrm{~cm} \times 4 \mathrm{~cm} \times 4 \mathrm{~cm}$ minimally enhancing hypodense lesion in the left adrenal gland with surrounding infiltrative changes (Fig). The patient underwent fluid resuscitation and was prescribed piperacillin/tazobactam. Retroperitoneal CT-guided drainage of the left adrenal collection performed the next day yielded $6 \mathrm{~mL}$ of pus. The patient also reported right knee pain for 1 week.
Physical examination revealed a right knee effusion. Right knee tapping yielded straw-coloured joint fluid, urgent Gram stain of which showed grampositive cocci. Emergency right knee arthroscopy with synovectomy was performed for septic arthritis. Intraoperatively there was pus within the knee joint. Adrenal fluid aspirate, joint fluid and blood culture all grew Streptococcus pyogenes (sensitive to penicillin). However, his swinging fever persisted. Extensive workup for other septic foci, including urine and sputum culture, nasal swab for viruses and bone marrow examination were all negative as were autoimmune markers and hepatitis and human immunodeficiency virus serology. He later developed sepsis-induced congestive heart failure with acute pulmonary oedema. Echocardiogram showed no valvular vegetation. The patient's haemodynamics gradually improved with antibiotics.

Sepsis again worsened with septic shock and desaturation. New chest radiograph revealed pulmonary congestion and a further CT scan showed interval shrinkage of the left adrenal abscess $(3.2 \mathrm{~cm} \times 2.9 \mathrm{~cm} \times 2.6 \mathrm{~cm})$. The left adrenal abscess was suspected as the remaining septic focus. Image-

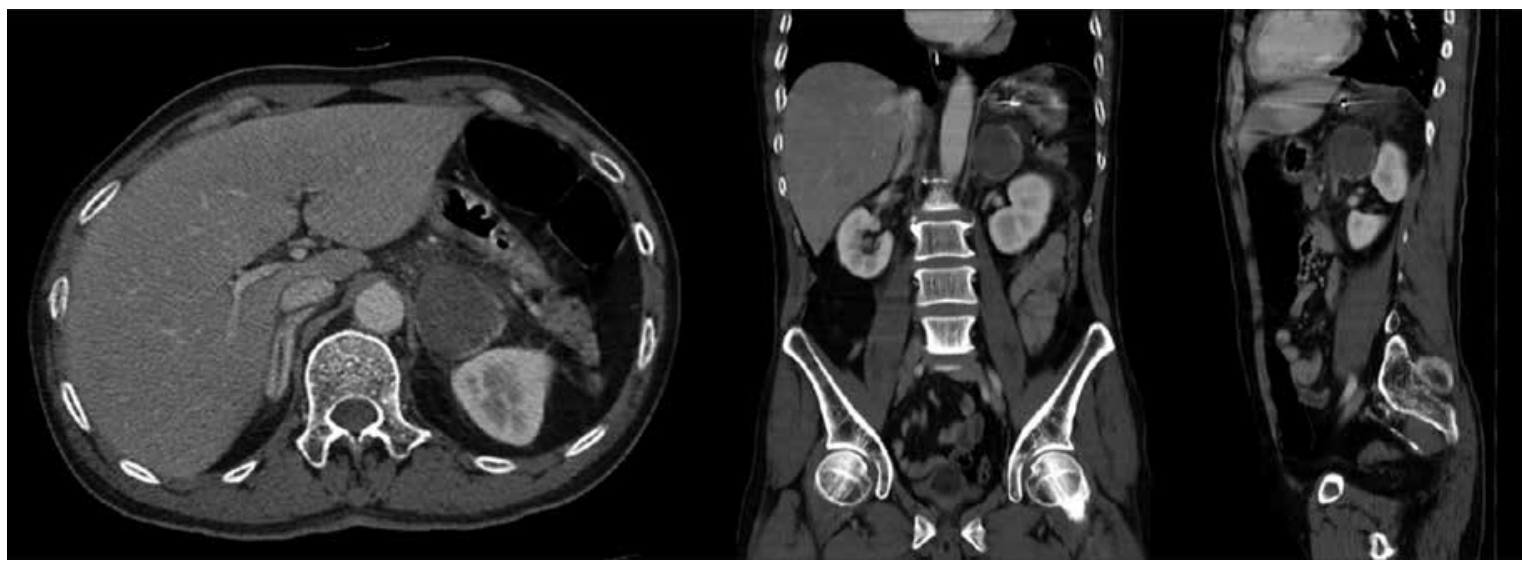

FIG. Axial, coronal, and sagittal computed tomography images showing left adrenal abscess 
guided drainage was not possible since there was no safety window. The abscess was small and very close to the aorta so emergency single-port laparoscopic left partial adrenalectomy was performed for definitive drainage.

The patient underwent general anaesthesia and was placed in a right lateral decubitus position. We adopted a transperitoneal approach with a 3-cm left subcostal incision. An OCTO-port was inserted, and pneumoperitoneum was established. Laparoscopy showed hyperaemic and inflammatory adhesions around the left adrenal gland. Splenic flexure was then taken down. The spleen and anterior surface of the left kidney were mobilised to enable a clearer view of the left adrenal gland. Initial tapping yielded no pus so the left adrenal gland was opened and the abscess cavity entered. Pus was drained and the cavity irrigated with normal saline. The superficial part of the abscess and its overlying adrenal tissue were then resected. A 15-French silicon drain was placed at the left adrenal bed. Finally, the fascial defect and skin were closed with an interrupted 2-0 absorbable suture and skin staples, respectively. The operating time was 210 minutes. Interval CT on postoperative day 4 showed disappearance of the left adrenal abscess. The surgical drain was removed on day 5. Private positron emission tomography-CT scan on day 15 showed no adrenal collection. The patient was discharged on postoperative day 33 . Histopathology was reported as abscess.

\section{Discussion}

To the best of our knowledge, this is the first case of adrenal abscess treated by emergency singleport laparoscopic partial adrenalectomy. Adrenal abscesses are uncommon in neonates and much rarer in adults. In the literature, most adult cases are due to disseminated infection in an immunocompromised host or postoperative complications. One rare case of acute appendicitis complicating adrenal abscess has been reported. ${ }^{1}$ Iatrogenic cause due to fine needle aspiration of an adrenal nodule has also been reported once only. ${ }^{2}$ Identified pathogens include Escherichia coli, Mycobacterium tuberculosis, Nocardia, Salmonella, and Streptococcus spp. Invasive group A streptococcus causing abdominal or peritoneal infections is rare. ${ }^{3}$ Adrenal abscess can present as fever, malaise, abdominal, or back pain. Diagnosis is by imaging, preferably CT. Principles of management are adequate drainage and systemic antimicrobials. Percutaneous image-guided drainage is useful for small and simple abscesses. Those that are large, multiloculated, lacking a safety window for needle insertion or in which percutaneous drainage has failed should be treated with surgical drainage.

After the first single-port adrenalectomy reported in 2005, there has been increasing evidence that laparo-endoscopic single-site adrenalectomy is safe and has a comparable surgical outcome to a conventional approach but with less wound pain and better cosmesis. ${ }^{4}$ Partial adrenalectomy has been applied for aldosterone-producing adenoma but is more challenging than total adrenalectomy as it is more difficult to gain a negative margin, ensure haemostasis, and minimise damage to the remaining adrenal tissue. ${ }^{5}$ The first single-port partial adrenalectomy was reported in 2010. Currently there is no solid evidence suggesting the best approach of surgery. In our centre, we performed our first singleport adrenalectomy in 2009. Since then, we routinely perform this technique for left-sided adrenal lesions and the surgical approach is transperitoneal.

Emergency adrenalectomy is much less well investigated. The most common indication is pheochromocytoma multisystem crisis, an acute severe presentation of catecholamine-induced hemodynamic instability causing end-organ damage or dysfunction. ${ }^{6}$ Emergency surgery is the treatment of choice, but the definitive timing of surgery is controversial.

Most reported cases that require surgical drainage have been treated by open surgery, ${ }^{7}$ except two cases where standard laparoscopic drainage was performed. Our patient is the first case of adrenal abscess treated by emergency single-port laparoscopic partial adrenalectomy. Intraoperatively, initial tapping of the abscess yielded no pus. Hence, we decided to proceed with partial adrenalectomy, to resect as little overlying adrenal tissue as possible, to drain the abscess adequately. At the end of surgery when inserting the silicon drain, we did not insert the drain via the same abdominal fascial defect at the OCTO-port to prevent incisional hernia. The drain exited the fascia adjacent to the fascial defect of the OCTO-port, then exited the skin at the same port site wound via a subcutaneous tunnel. There were no surgical complications and postoperative recovery was smooth.

In summary, adrenal abscess is rare in healthy adults but can cause severe sepsis. Prompt surgery is important when conservative management fails. We demonstrate that emergency single-port laparoscopic partial adrenalectomy is a feasible approach for surgical drainage. More large-scale studies and randomised trials are needed to provide more solid evidence to support use of single-port adrenalectomy in an emergency setting.

\section{Author contributions}

All authors contributed to the concept or design of the study, acquisition of the data, analysis or interpretation of the data, drafting of the manuscript, and critical revision of the manuscript for important intellectual content. All authors had full access to the data, contributed to the study, approved the final version for publication, and take responsibility for its accuracy and integrity. 


\section{Conflicts of interest}

All authors have disclosed no conflicts of interest.

\section{Funding/support}

This case report received no specific grant from any funding agency in the public, commercial, or not-for-profit sectors.

\section{Ethics approval}

The patient was treated in accordance with the Declaration of Helsinki. The patient gave written informed consent for all investigations and interventions.

\section{References}

1. Dimofte G, Dubei L, Lozneanu LG, Ursulescu C, Grigora Scedil M. Right adrenal abscess-an unusual complication of acute appendicitis. Rom J Gastroenterol 2004;13:241-4

2. Masmiquel L, Hernandez-Pascual C, Simo R, Mesa J.
Adrenal abscess as a complication of adrenal fine-needle biopsy. Am J Med 1993;95:244-5.

3. Nelson GE, Pondo T, Toews KA, et al. Epidemiology of invasive group A Streptococcal infections in the United States, 2005-2012. Clin Infect Dis 2016;63:478-86.

4. Chung SD, Huang CY, Wang SM, Tai HC, Tsai YC, Chueh SC. Laparoendoscopic single-site (LESS) retroperitoneal adrenalectomy using a homemade singleaccess platform and standard laparoscopic instruments. Surg Endosc 2011;25:1251-6.

5. Yu CC, Tsai YC. Current surgical technique and outcomes of laparoendoscopic single-site adrenalectomy. Urol Sci 2017;28:59-62.

6. Wu R, Tong N, Chen X, et al. Pheochromocytoma crisis presenting with hypotension, hemoptysis, and abnormal liver function: a case report. Medicine (Baltimore) 2018;97:e11054.

7. Jackson C, McCullar B, Joglekar K, Seth A, Pokharna H. Disseminated nocardia farcinica pneumonia with left adrenal gland abscess. Cureus 2017;9:e1160. 